\title{
A Erosividade das Chuvas: o Manejo Agrícola Sustentável das Terras do Município de Castanhal/PA
}

\author{
José Albuquerque ${ }^{1}$, Jessivaldo Rodrigues Galvão ${ }^{1}$, Maria Helena Moraes ${ }^{2}$, Mateus Augusto de Carvalho Santana ${ }^{1}$, \\ Leonardo de Almeida Oliveira ${ }^{1}$ \& Mateus Correia Lima ${ }^{1}$
}

Recebido em 28/09/2020 - Aceito em 15/03/2021

1 Universidade Federal Rural da Amazônia, Instituto ICA, Departamento de Ciências Agrárias, Belém/PA, Brasil. CEP: 66.077-830. <albuqprof@yahoo.com.br, jessigalvao50@gmail.com, ma1041578@gmail.com, leonardoaaloliveira@gmail.com, mabone10@gmail.com>.

2 Universidade Estadual Paulista, Instituto FCA, Departamento de Ciências Agrárias, São Paulo/SP, Brasil. CEP: 18610-034. $<$ mhmoraes@fca.unesp.br>.

RESUMO - Com vistas ao planejamento agrícola sustentável, foi determinada a erosividade das chuvas do município de Castanhal, localizado na região nordeste do estado do Pará. Foi utilizada uma série contínua de 39 anos de registros pluviométricos para cálculo dos coeficientes de chuva, os quais foram utilizados na determinação da erosividade das chuvas através de equação ajustada com as condições pluviométricas de Belém, com disponibilidade de dados pluviográficos, com semelhança climática e distribuição de chuvas verificada através de análise de correlação $(r=0,97)$. A erosividade média anual para a localidade estudada foi estimada em $22001 \mathrm{MJmm} \cdot \mathrm{ha}^{-1} \cdot \mathrm{h}^{-1}$. ano ${ }^{-1}$, com probabilidade de retorno de $49,6 \%$ a cada 2,02 anos. Os valores de retorno para os períodos de 5, 10, 20, 50 e 100 anos foram 24162, 25908, 27601, 29782 e 31406 MJmm.ha' ${ }^{-1} \cdot h^{-1}$.ano-1, respectivamente.

Palavras-chave: Agricultura sustentável; bacia hidrográfica; conservação do solo, erosão hídrica; perda de solo.

\section{The Erosivity of Rains: Sustainable Agricultural Management of the Lands of the Municipality of Castanhal/PA}

ABSTRACT - Aiming at sustainable agricultural planning, the rain erosivity of the municipality of Castanhal, located in the northeast region of the state of Pará, was determined. A continuous series of 39 years of rainfall records was used to calculate the rain coefficients, which were used in determining of rainfall erosivity through an equation adjusted to the rainfall conditions of Belém, with availability of pluviographic data, with climatic similarity and rainfall distribution verified through correlation analysis $(r=0.97)$. The average annual erosivity for the studied location was estimated at 22001 MJmm. ha ${ }^{-1} \cdot \mathrm{h}^{-1}$. year $^{-1}$, with a probability of return of $49.6 \%$ every 2.02 years. The return values for the periods of 5, 10, 20, 50 and 100 years were 24162, 25908, 27601, 29782 and $31406 \mathrm{MJmm} . \mathrm{ha}^{-1} \cdot \mathrm{h}^{-1}$. year ${ }^{-1}$, respectively.

Keywords: Sustainable agriculture; soil conservation; soil losses; sustainable agriculture, water erosion, watershed.

\section{Erosividad de la Lluvia: Gestión Sostenible de Tierras Agrícolas en el Municipio de Castanhal/PA}

RESUMEN - Con el objetivo de una planificación agrícola sostenible, se determinó la erosividad pluvial del municipio de Castanhal, ubicado en la región nororiental del estado de Pará. Se utilizó una serie continua de 39 años de registros pluviométricos para calcular los coeficientes de lluvia, los cuales se utilizaron en la determinación de la erosividad pluvial mediante una ecuación ajustada a las condiciones pluviales en Belém, con disponibilidad de datos pluviográficos, con similitud climática y distribución pluvial verificada mediante análisis de correlación $(\mathrm{r}=0,97)$. La erosividad promedio anual para la ubicación estudiada se estimó en $22001 \mathrm{MJmm} \cdot \mathrm{ha}^{-1} \cdot \mathrm{h}^{-1}$.year ${ }^{-1}$, con una probabilidad de retorno del $49.6 \%$ cada 2.02 años. Los valores de retorno para los períodos de 5, 10, 20, 50 y 100 años fueron 24162, 25908, 27601, 29782 y $31406 \mathrm{MJmm} \cdot \mathrm{ha}^{-1} \cdot \mathrm{h}^{-1}$.year ${ }^{-1}$, respectivamente.

Palabras clave: Agricultura sostenible; cuenca hidrográfica; conservación de suelos, erosión hídrica; pérdida de suelo. 


\section{Introdução}

A Amazônia é mundialmente conhecida como a região das florestas tropicais densas. É também a região das águas, armazenando em sua complexa hidrografia cerca de 1/5 da água doce do planeta. Entretanto, seus solos são bastante erodíveis, de baixa fertilidade natural em grande parte, $e$ as áreas exploradas com agricultura estão submetidas a elevados índices pluviométricos, condições essas que favorecem a erosão hídrica, necessitando em vários casos de um planejamento racional para o uso agrícola sustentável dessas áreas. A determinação da erosividade da chuva possibilita identificar, durante o ano, os meses nos quais há maior risco de perda de solo $e$ água, informação que tem papel fundamental no planejamento conservacionista urbano e rural em épocas de maior capacidade erosiva das chuvas (Bertoni \& Lombardi Neto, 1990; Silva et al., 2010).

A erosão hídrica é causada pelo impacto das gotas de chuvas que promovem a desagregação de partículas do solo que, posteriormente, são transportadas pelo escoamento superficial. Conhecer o potencial erosivo das chuvas é crucial para estimar a perda de solo (Shamshad et al., 2008; Carvalho et al., 2014). Entendendo-se isso, envolve inevitavelmente a necessidade de adoção de práticas de conservação do solo $e$ água nas glebas cultivadas, tendo em vista que o destino final dos materiais sólidos erosionados, fertilizantes e agrotóxicos são os mananciais aquíferos, que compõem a bacia hidrográfica sob a influência das chuvas locais causando grande impacto ambiental.

Para estudar a produção de sedimentos, alguns modelos determinam o poder erosivo das chuvas. Os modelos mais utilizados para estimar a produção de sedimentos por erosão laminar são a Equação Universal de Perda de Solo (Universal Soil Loss Equation - USLE) (Wischmeier \& Smith, 1978) e a Equação Universal de Perda de Solo Modificada (Modified Universal Soil Loss Equation - MUSLE) (Williams, 1975). A MUSLE é uma versão modificação da USLE, desenvolvida para estimar a produção de sedimentos em eventos chuvosos em uma bacia, diferentemente da USLE, que estima a perda de solo média, não levando em consideração o transporte de sedimentos.

A equação universal de perda de solo (USLE) é um modelo matemático que pode ser usado para predizer as perdas de solo susceptíveis de ocorrer numa área de exploração agrícola, de forma que a magnitude dessas perdas fique situada dentro de limites toleráveis. Essa equação relaciona os fatores que influenciam no processo da erosão hídrica do solo, assim descritos: erosividade das chuvas (R); erodibilidade do solo (K); comprimento e grau de declive do terreno (LS); cobertura e manejo do solo (C) e práticas conservacionistas $(\mathrm{P})$. A análise preliminar dessa equação é de fundamental importância, como subsídio, para aplicação do manejo e práticas mais adequadas, visando um maior controle de perdas de solo por erosão hídrica. Nas equações (USLE) e a sua versão modificada (MUSLE), o poder erosivo da chuva é explicado pelo fator de erosividade $(\mathrm{R})$, que combina os efeitos da duração, magnitude e intensidade de cada evento chuvoso (Wischmeier \& Smith, 1978; Damé et al., 2012).

Dentre os fatores da equação de perdas de solo, destaca-se a erosividade das chuvas (R), ressaltando-se que a determinação desse fator independe dos demais, necessitando para isso de um levantamento de dados para caracterização do regime pluviométrico da região estudada, através da coleta de informações pluviográficas obtidas em estações meteorológicas, dados esses raros e de difícil acesso na maior parte dos casos, e de análise extremamente exaustiva. Diante disso, autores diversos tem feito uso de equações ajustadas entre o índice de erosividade (R), obtidos com dados pluviográficos, e os coeficientes de chuva (Rc), conhecido como o índice de Fournier, o qual é calculado somente com informações pluviométricas da mesma localidade. Essas equações ajustadas podem ser utilizadas para estimar a erosividade das chuvas de outras regiões, com semelhança de distribuição da precipitação pluviométrica e clima, em relação à localidade para a qual a equação foi ajustada.

O objetivo deste estudo foi avaliar a erosividade das chuvas, períodos e probabilidades de retorno, no município de Castanhal, localizado na região nordeste do estado do Pará, visando o uso e manejo agrícola sustentável das terras do município.

\section{Material e Métodos}

O trabalho foi realizado a partir de dados pluviométricos, registrados no município de 
Castanhal, estado do Pará, obtidos na EmbrapaAmazônia Oriental (de 1973 a 1987) e no Centro de Hidrologia da Companhia de Pesquisa de Recursos minerais (CPRM) (de 1988 a 2011), perfazendo uma série histórica contínua de 39 anos de precipitação pluviométrica.

O clima regional, segundo a classificação de Koppen, é Ami. As temperaturas máxima e mínima são de 35 e $18^{\circ} \mathrm{C}$, respectivamente, com temperatura média anual de $26^{\circ} \mathrm{C}$. A umidade relativa média e a precipitação anual são de $80 \%$ e 2.571,6mm, respectivamente e (Tenório et al., 1999; Souza, 2017).

Foram analisados os dados mensais $e$ anuais de precipitação pluviométrica da série contínua de 39 anos, correspondente ao período de 1973 a 2011, utilizando-se planilha eletrônica para tabulação dos dados, obtendo-se as médias mensais e anuais de precipitação para toda a série histórica.

Tendo em vista a inexistência de registros pluviográficos no município, a erosividade das chuvas foi determinada através dos coeficientes de chuva (Rc) de Fournier, calculados pela seguinte equação:

$$
\mathrm{Rc}=\frac{\mathrm{p}^{2}}{\mathrm{P}}
$$

Em que:

$\mathrm{Rc}=$ coeficiente de chuva

$\mathrm{p}=$ precipitação mensal

$\mathrm{P}=$ precipitação anual.

$\mathrm{Na}$ determinação da erosividade das chuvas, foi utilizada a seguinte equação ajustada por Oliveira Junior et al. (1994) para as condições climáticas de Belém, com significativo coeficiente de correlação linear $(r=0,99)$.

$$
\mathrm{EI}_{30}=753,11+44,74 \mathrm{Rc}
$$

Em que:

$\mathrm{EI}_{30}=$ erosividade

$\mathrm{Rc}=$ coeficiente de chuva.

De acordo com o que propõe Renard \& Freimund (1994), os coeficientes de chuva foram calculados mês a mês para toda a série histórica. A erosividade mensal foi calculada com base na Equação 1. Obtendo-se os valores de erosividade das chuvas para cada ano, por meio da soma das erosividades mensais. Foi determinada a média anual representativa da erosividade nessa região, pela média das erosividades anuais, no período de 39 anos.

A possibilidade de extrapolação da equação de ajuste, de acordo com a Equação 2, foi previamente verificada levando-se em consideração a semelhança climática entre Belém e Castanhal, ambas com o mesmo tipo climático, bem como as semelhanças das curvas de distribuição das precipitações mensais, verificadas pela análise de correlação, obtendo-se uma significativa correlação linear entre as distribuições de chuvas (Figuras 1 e 2), o que possibilitou com grande margem de confiabilidade o uso da equação de ajuste.

As análises dos períodos de retorno, das probabilidades de ocorrência e dos índices de erosividades das chuvas, foram efetuadas utilizando-se a lei da probabilidade logarítmica e a teoria dos valores extremos, preconizadas por Schwab et al. (1981), através das seguintes equações:

$$
\begin{aligned}
& \mathrm{T}=\frac{(\mathrm{N}+1)}{\mathrm{n}} \\
& \operatorname{Pr}=\frac{100}{\mathrm{~T}} \\
& \mathrm{X}_{\mathrm{C}}=\mathrm{X}(1+\mathrm{CV} \cdot \mathrm{k}) \\
& \mathrm{K}=\frac{(-2,45 \cdot(\mathrm{y}+\ln (\ln \mathrm{T}-\ln (\mathrm{T}-1)))}{\mathrm{pi}}
\end{aligned}
$$

Em que:

$\mathrm{T}=$ tempo de retorno em anos

$\mathrm{N}=$ número de anos da série

$\mathrm{n}=$ número de ordem quando os valores são relacionados em ordem decrescente

$\operatorname{Pr}=$ probabilidade de ocorrência do evento $(\%)$

$\mathrm{X}_{\mathrm{C}}=$ estimativa do valor teórico do evento

$\mathrm{X}=$ média dos índices de erosividades

$\mathrm{CV}=$ coeficiente de variação dos índices de erosividades

$\mathrm{k}$ = fator de frequência para determinado período

$\lambda=$ constante de Euler $(0,577)$

Para determinação da série histórica mínima adequada para determinação da erosividade, 
foi utilizada a equação preconizada por Ribeiro Junior (2001):

$$
\mathrm{N}=\frac{\left(\mathrm{CV}^{2}+12\right)}{(\mathrm{E})}
$$

Em que:

$\mathrm{N}=$ número mínimo de anos

$\mathrm{CV}=$ coeficiente de variação

$\mathrm{t}=$ valor estatístico de Student $(\mathrm{p}=95 \%)$

$\mathrm{E}=$ fração de erro da média $(10 \%)$

\section{Resultados e Discussão}

A Figura 1 ilustra as distribuições mensais de chuvas de Belém e Castanhal, mostrando dados semelhantes de pluviosidade no período avaliado.

A fim de verificar a possibilidade de uso da Equação 5, ajustada para as condições pluviométricas de Belém, foi procedida a análise de correlação de Pearson entre os dados médios mensais de distribuição de chuva de Belém (variável independente) e os dados de chuva da

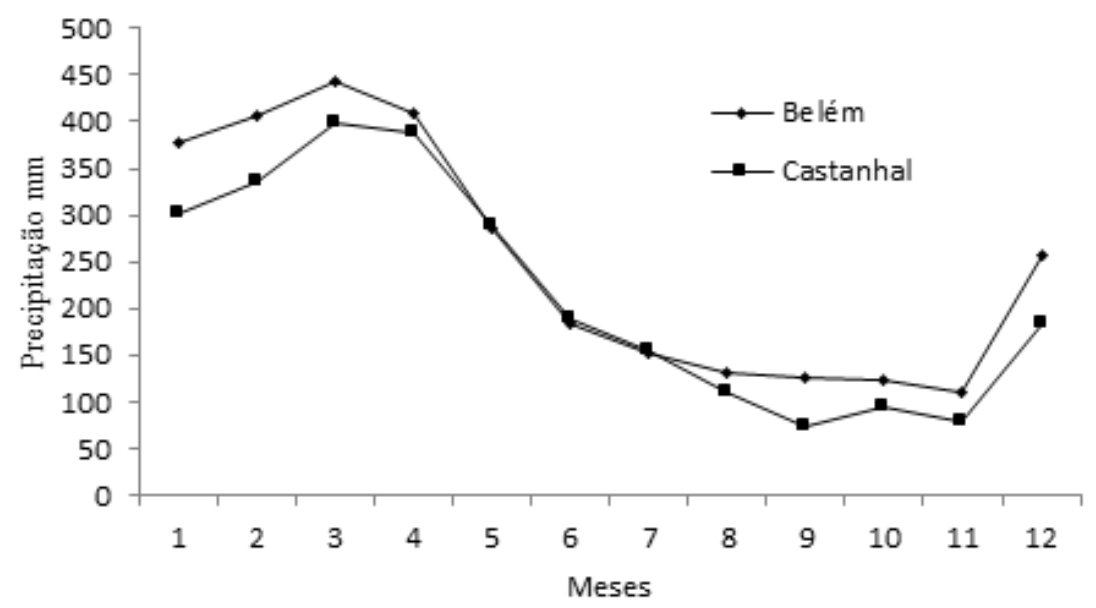

Figura 1 - Distribuição mensal das precipitações de Belém e Castanhal (bacia do rio Apeú).

área da bacia do rio Apeú (variável dependente), dados esses apresentados na Tabela 1.
As ocorrências mensais de chuva correlacionadas entre as duas localidades mostram indícios

Tabela 1 - Distribuição de chuvas das localidades para análise de semelhança.

\begin{tabular}{|c|c|c|}
\hline \multirow{2}{*}{ Mês } & \multicolumn{2}{|c|}{ Precipitação pluviométrica (mm) } \\
\cline { 2 - 3 } & Belém & Bacia do rio Apeú \\
\hline Jan & 378,5 & 302,6 \\
\hline Fev & 406,7 & 334,5 \\
\hline Mar & 444,1 & 398,1 \\
\hline Abr & 408,9 & 387,0 \\
\hline Mai & 286,4 & 289,3 \\
\hline Jun & 182,3 & 188,7 \\
\hline Jul & 151,6 & 155,3 \\
\hline Ago & 130,5 & 111,4 \\
\hline Set & 127,0 & 74,5 \\
\hline Out & 122,4 & 95,0 \\
\hline Nov & 111,4 & 79,8 \\
\hline Dez & 257,2 & 182,5 \\
\hline Total & $\mathbf{3 0 0 7}$ & $\mathbf{2 5 9 9}$ \\
\hline
\end{tabular}


de semelhança nas distribuições, comprovada pela análise de correlação de Pearson (Tabela 2), tendo sido encontrado um coeficiente de correlação linear muito significativo ( $r=0,97)$. Moreti et al. (2003) utilizou a intercessão de intervalos de confiança em torno da média de precipitações pluviométricas, para avaliar a semelhança de distribuições de chuva. Mello et al. (2007) consideraram apenas a semelhança do tipo climático, como na maior parte dos casos, para extrapolar o uso de equações de ajustes, de uma localidade para outra, na determinação da erosividade das chuvas.

Tabela 2 - Correlação de Pearson entre as precipitações mensais de Belém e Castanhal.

\begin{tabular}{|c|c|c|c|c|c|}
\hline Variável & Variável & Observações & Correlação & T & Significância \\
\hline Belém & Castanhal & 12 & 0.9728 & 13.2750 & 0.0000 \\
\hline
\end{tabular}

Constatou-se que a análise da correlação realizada, possibilitou estimar a erosividade das chuvas do município de Castanhal, de acordo com a equação gerada (Fig. 2).

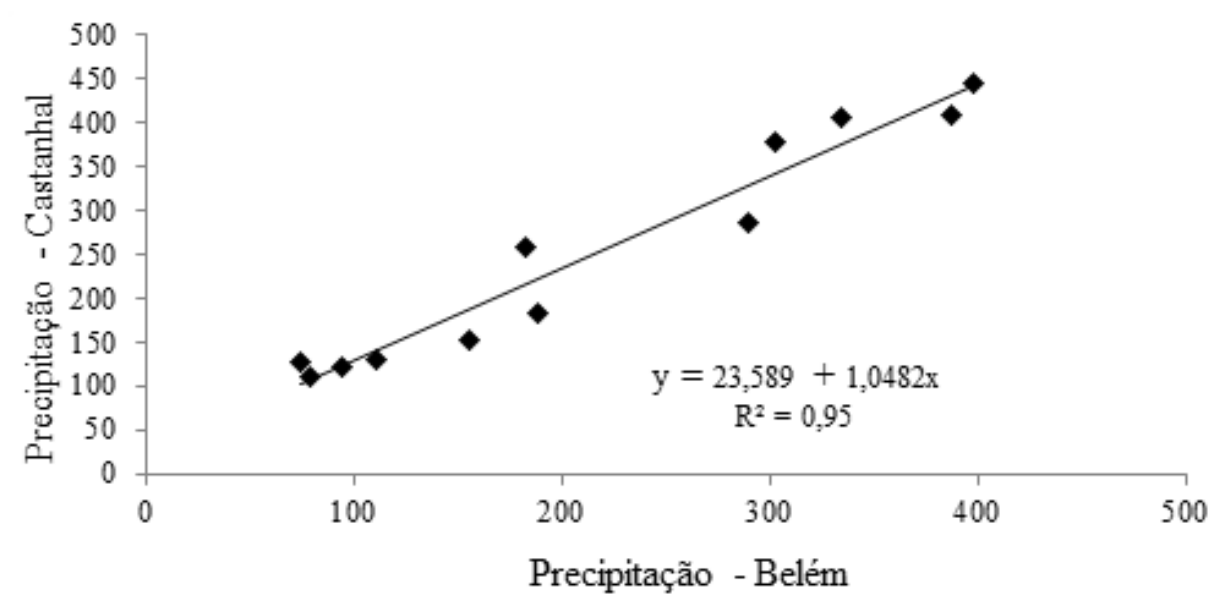

Figura 2 - Correlação entre as distribuições de chuvas de Belém e Castanhal (bacia do Rio Apeú).

Os dados referentes à erosividade indicam média de $22.001 \mathrm{MJmm} \cdot \mathrm{ha}^{-1} \cdot \mathrm{h}^{-1}$. ano ${ }^{-1}$, que são considerados valores muito altos (Tabela 3). Estes valores podem ter sido ocasionados pela alta pluviosidade, no período, e as características arenosas do solo. Segundo Foster et al. (1981), erosividades das chuvas com valores a partir de $8.000 \mathrm{MJmm} \cdot \mathrm{ha}^{-1} \cdot \mathrm{h}^{-1}$.ano ${ }^{-1}$, são classificadas como muito altas. Oliveira Junior (1992), citado por Oliveira Junior et al. (1994), encontrou para o município de Bragança, região nordeste do Pará (clima tipo Am), um valor de erosividade anual de 12.350 MJmm.ha- ${ }^{-1} \cdot \mathrm{h}^{-1} \cdot$ ano $^{-1}$, e para o município de Marabá, região sul do Pará (clima Aw), de 13.914 MJmm.ha- ${ }^{-1} \cdot h^{-1} \cdot$ ano $^{-1}$.
A erosividade média anual (Tabela 3), foi estimada em $22.001 \mathrm{MJmm} \cdot \mathrm{ha}^{-1} \cdot \mathrm{h}^{-1} \cdot \mathrm{ano}^{-1}$, que representa o valor " $R$ " da equação universal de perda de solo (USLE), assim descrita: $\mathrm{A}=\mathrm{R} . \mathrm{K}$. (L.S).(C.P)) em que $A$ = perda média anual de solo por unidade de área (ton/ha.ano); $\mathrm{R}=$ erosividade da chuva (Mj.mm/ha.h.ano); $\mathrm{K}=$ erodibilidade do solo; $\mathrm{L}=$ comprimento da vertente; $\mathrm{S}=$ declividade da vertente; $\mathrm{C}=$ uso e manejo do solo; e $\mathrm{P}=$ práticas conservacionistas. Os valores variaram de 17.299 a $31.452 \mathrm{MJmm} \cdot \mathrm{ha}^{-1} \cdot \mathrm{h}^{-1}$.ano ${ }^{-1}$, com um coeficiente de variação de $13,64 \%$, com os respectivos valores teóricos do tempo de retorno e probabilidades de ocorrência. 
Tabela 3 - Valores médios mensais de precipitação, coeficientes de chuva e índices de erosividades, no período de 1973 a 2011.

\begin{tabular}{|l|c|c|c|}
\hline \multirow{2}{*}{ Mês } & Precipitação pluviométrica & Coeficiente de chuva & Erosividade $\mathbf{( E I}_{\mathbf{3 0}} \mathbf{)}$ \\
\cline { 2 - 4 } & $\mathbf{m m}$ & $\mathbf{m m}$ & $\mathbf{M J m m}_{\mathbf{h}} \mathbf{h a}^{\mathbf{- 1}} \mathbf{h}^{\mathbf{1}}$ \\
\hline Janeiro & 302,6 & 39,8 & $2.414,7$ \\
\hline Fevereiro & 334,5 & 48,2 & $2.766,1$ \\
\hline Março & 398,1 & 65,3 & $3.479,2$ \\
\hline Abril & 387,0 & 62,1 & $3.343,1$ \\
\hline Maio & 289,3 & 36,0 & $2.254,5$ \\
\hline Junho & 188,7 & 15,2 & $1.386,4$ \\
\hline Julho & 155,3 & 10,6 & $1.197,4$ \\
\hline Agosto & 111,4 & 5,6 & 985,5 \\
\hline Setembro & 74,5 & 2,9 & 873,4 \\
\hline Outubro & 95,0 & 5,5 & 981,3 \\
\hline Novembro & 79,8 & 4,1 & 922,9 \\
\hline Dezembro & 182,5 & 15,4 & $1.396,8$ \\
\hline Total & $\mathbf{2 . 5 9 8 , 8}$ & & $\mathbf{2 2 . 0 0 1 , 3}$ \\
\hline
\end{tabular}

Analisando os valores relativos da erosividade mensal entre os anos de 1973 a 2011, observa-se que no primeiro semestre do período (janeiro a junho) ocorre $71,1 \%$ da erosividade anual, sendo os meses de fevereiro, março, abril e maio os de maiores recorrências, valor compatível com a distribuição da precipitação na região, indicando que neste período é provável que tenha ocorrido a maior parte das perdas anuais de solo por erosão em Castanhal. Apesar das variações nos valores mensais da precipitação e suas características possam explicar grande parte das variações na erosividade (Bazzano et al., 2010), vale destacar que estes não são os únicos fatores que influenciam os processos erosivos, em que podemos citar a geologia, pedologia, relevo e o uso do solo (Silva \& Machado, 2014).

Foi registrado o período de retorno (Tabela 4), que é definido como o intervalo de tempo em anos, dentro do qual determinado evento hidrológico máximo poderá ser igualado ou ultrapassado, sendo outro importante parâmetro relacionado com as chuvas. Dessa forma, os valores máximos anuais aproximamse de um padrão definido de distribuição de frequência, quando o número de observações se torna o mais amplo possível (Schwab et al., 1981).

Tabela 4 - Período de retorno e probabilidade de ocorrência dos valores anuais de erosividade $\left(\mathrm{EI}_{30}\right)$ na bacia hidrográfica do Rio Apeú.

\begin{tabular}{|c|c|c|c|c|}
\hline \multirow{2}{*}{ Ano } & Erosividade $\left(\mathrm{EI}_{30}\right)$ & Número de ordem (n) & Período de retorno $(\mathrm{T})$ & Probabilidade (Pr) \\
\hline & MJmm.ha $a^{-1} \cdot \mathbf{h}^{-1} \cdot$ ano $^{-1}$ & & Ano & $\%$ \\
\hline 2001 & $31.451,80$ & 1 & 40,00 & 2,5 \\
\hline 1985 & $27.895,70$ & 2 & 20,00 & 5,0 \\
\hline 1974 & $26.633,10$ & 3 & 13,33 & 7,5 \\
\hline 1988 & $24.916,40$ & 4 & 10,00 & 10,0 \\
\hline 1980 & $24.867,50$ & 5 & 8,00 & 12,5 \\
\hline 1975 & $24.724,90$ & 6 & 6,67 & 15,0 \\
\hline
\end{tabular}




\begin{tabular}{|c|c|c|c|c|}
\hline 2009 & $24.617,20$ & 7 & 5,71 & 17,5 \\
\hline 1992 & $24.186,50$ & 8 & 5,00 & 20,0 \\
\hline 1991 & $24.037,90$ & 9 & 4,44 & 22,5 \\
\hline 2002 & $23.991,70$ & 10 & 4,00 & 25,0 \\
\hline 1989 & $23.862,60$ & 11 & 3,64 & 27,5 \\
\hline 1995 & $23.811,50$ & 12 & 3,33 & 30,0 \\
\hline 1994 & $23.452,30$ & 13 & 3,08 & 32,5 \\
\hline 2004 & $22.942,30$ & 14 & 2,86 & 35,0 \\
\hline 1997 & $22.771,80$ & 15 & 2,67 & 37,5 \\
\hline 1976 & $22.553,40$ & 16 & 2,50 & 40,0 \\
\hline 1986 & $22.526,00$ & 17 & 2,35 & 42,5 \\
\hline 1982 & $22.492,60$ & 18 & 2,22 & 45,0 \\
\hline 1984 & $22.094,30$ & 19 & 2,10 & 47,5 \\
\hline 1990 & $21.556,50$ & 20 & 2,00 & 50,0 \\
\hline 2005 & $21.431,00$ & 21 & 1,90 & 52,5 \\
\hline 1998 & $21.178,90$ & 22 & 1,82 & 55,0 \\
\hline 1973 & $21.064,80$ & 23 & 1,74 & 57,5 \\
\hline 1978 & $21.047,40$ & 24 & 1,67 & 60,0 \\
\hline 1999 & $20.897,90$ & 25 & 1,60 & 62,5 \\
\hline 2006 & $20.711,80$ & 26 & 1,54 & 65,0 \\
\hline 1987 & $20.704,50$ & 27 & 1,48 & 67,5 \\
\hline 1977 & $20.123,80$ & 28 & 1,43 & 70,0 \\
\hline 2008 & $20.050,50$ & 29 & 1,38 & 72,5 \\
\hline 2011 & $19.919,90$ & 30 & 1,33 & 75,0 \\
\hline 1993 & $19.816,90$ & 31 & 1,29 & 77,5 \\
\hline 2010 & $19.367,70$ & 32 & 1,25 & 80,0 \\
\hline 2007 & $18.818,40$ & 33 & 1,21 & 82,5 \\
\hline 2000 & $18.582,70$ & 34 & 1,18 & 85,0 \\
\hline 1983 & $18.219,30$ & 35 & 1,14 & 87,5 \\
\hline 1981 & $17.923,60$ & 36 & 1,11 & 90,0 \\
\hline 2003 & $17.765,50$ & 37 & 1,08 & 92,5 \\
\hline 1979 & $17.739,60$ & 38 & 1,05 & 95,0 \\
\hline 1996 & $17.298,60$ & 39 & 1,03 & 97,5 \\
\hline MÉDIA & $22.001,30$ & & & \\
\hline CV (\%) & 13,64 & & & \\
\hline
\end{tabular}

Os valores das erosividades mensais (Tabela 5) apresentaram coeficientes de variação de 15,41 a $59,15 \%$, nos meses de setembro e maio, respectivamente, o que pode ter relação com a proximidade do início do período menos chuvoso e o final da estiagem, meses esses mais sujeitos às variações de ocorrências de chuvas. 
Tabela 5 - Distribuição mensal dos valores absolutos e relativos da erosividade das chuvas na bacia hidrográfica do Rio Apeú.

\begin{tabular}{|c|c|c|c|}
\hline \multirow{2}{*}{ Mês } & \multicolumn{2}{|c|}{ Erosividade das chuvas } & \multicolumn{1}{c|}{ CV } \\
\cline { 2 - 4 } & Absoluta & Relativa & \% \\
\hline Jan & ${\text { MJmm.ha-1. } \mathbf{h}^{-1}}^{*}$ & \% & 52,7 \\
\hline Fev & 2415 & 10,98 & 55,8 \\
\hline Mar & 2766 & 12,57 & 37,1 \\
\hline Abr & 3479 & 15,81 & 39,4 \\
\hline Mai & 3343 & 15,19 & 59,2 \\
\hline Jun & 2255 & 10,25 & 42,8 \\
\hline Jul & 1386 & 6,30 & 26,4 \\
\hline Ago & 1197 & 5,44 & 18,3 \\
\hline Set & 986 & 4,48 & 15,4 \\
\hline Out & 873 & 3,97 & 43,9 \\
\hline Nov & 981 & 4,46 & 30,9 \\
\hline Dez & 923 & 4,20 & 43,0 \\
\hline Total & 1397 & 6,35 & \\
\hline
\end{tabular}

Por meio da Equação 5, foram calculados os tempos de retorno das erosividades das chuvas para os períodos de 2, 5, 10, 20, 50 e 100 anos, representados na Tabela 6.

Tabela 6 - Erosividade $\left(\mathrm{EI}_{30}\right)$ de retorno para vários períodos.

\begin{tabular}{|c|c|c|}
\hline Tempo de retorno & Fator de frequência & Erosividade de retorno \\
\hline Ano & $\mathbf{k}$ & $\mathbf{M J m m}^{-h^{-1} \cdot \mathbf{h}^{-\mathbf{1}} \cdot \mathbf{a n o}^{-\mathbf{1}}}$ \\
\hline 2 & $-0,164$ & 21.517 \\
\hline 5 & $+0,720$ & 24.162 \\
\hline 10 & $+1,302$ & 25.908 \\
\hline 20 & $+1,866$ & 27.601 \\
\hline 50 & $+2,593$ & 29.782 \\
\hline 100 & $+3,134$ & 31.406 \\
\hline
\end{tabular}

Os valores teóricos das erosividades de retorno para os períodos de 2, 5, 10 e 20 anos aproximam-se bastante dos valores dos eventos já ocorridos. Isso se deve ao baixo valor do coeficiente de variação da erosividade média anual que podem ser observados na Tabela 4.

\section{Conclusões}

O período quadrimestral de janeiro a abril, por ser o de maior índice pluviométrico, é o mais suscetível à erosão hídrica dos solos, como mostram os índices mensais acumulados de erosividade das chuvas. 
A erosividade das chuvas no município de Castanhal é um fator muito relevante nas tomadas de decisões sobre as práticas de manejo do solo mais adequadas, visando à sustentabilidade da exploração agrícola.

Este estudo corresponde um aspecto essencial para o planejamento das atividades agrícolas, uma vez que o conhecimento de seus padrões espaciais e temporais possibilitam planejar as etapas do calendário agrícola e implantar práticas de manejo que minimizem os impactos das chuvas.

\section{Referências}

Bertoni, J \& Lombardi NF. Conservação do solo. São Paulo: Ícone; 1990.

Bazzano MGP, Eltz FLF \& Cassol EA. Erosividade e características hidrológicas das chuvas de Rio Grande (RS). R Bras Ci Solo, 34: 235-244, 2010.

Carvalho DF, Durigon VL, Antunes MAH, Almeida WS \& Oliveira PTS. Predicting soil erosion using Rusle and NDVI time series from TM Landsat 5. Pesquisa Agropecuária Brasileira, 49: 215-224, 2014.

Damé RCF, Teixeira CFA, Gonçalves T, Santos JP \& Quadro MS. Erosividade sob duas durações de intensidade máximas da chuva em Pelotas/RS. Revista Agro@mbiente, 6(1): 01-08, 2012.

Foster $\mathrm{G}$ et al. Conversion of the universal soil loss equation to metric units.Soil Water Conserv., 36: 355359, 1981.

Moreti $\mathrm{D}$ et al. Importantes características de chuva para a conservação do solo e da água no município de São Manuel/SP. Rev. Bras. Ci. Solo, 27(4): 1-14, 2003.

Mello $\mathrm{C}$ et al. Erosividade mensal e anual no estado de Minas Gerais. Pesq. agropec. Bras. Brasilia, 2(4): 537545, 2007.

Oliveira JR, Chaves R \& Melo A. A erosividade das chuvas em Belém/PA. Belém, Univ. Fed. Rural Amazônia, Boletim 22: 35-52, 1994.
Renard K \& Freimund J. Using monthly precipitation data to estimate the R-factor in the revised USLE. Jornal of Hidrology, 157: 287-306, 1994.

Ribeiro JJ. Análises estatísticas no SAEG. Viçosa, UFV, 301: 169-226, 2001.

Schwab G. Soil and water conservation engineering. New York, John Wiley \& Sons, 683p. 1981.

Shamshad et al. Rainfall erosivity map for Brazil. Catena, 57(3): 251-259, 2004.

Shamshad A, Azhari MN, Isa MH, Hussin WMA \& Parida BP. Development of an appropriate procedure for estimation of RUSLE EI30 index and preparation of erosivity maps for Pulau Penang in Peninsular Malaysia. Catena, 72: 423-432, 2008.

Silva MAS, Silva MLN, Curi N, Santos GR, Marques JJGSM \& Menezes MD. Avaliação e espacialização da erosividade da chuva no Vale do Rio Doce, Região Centro-Leste do estado de Minas Gerais. R Bras Ci Solo, 34: 1029-1039, 2010.

Silva VCB \& Machado PS. SIG na análise ambiental: suscetibilidade erosiva da bacia hidrográfica do Córrego Mutuca, Nova Lima - Minas Gerais. R Geogr, 31(2): 66-87, 2014.

Souza PJOP, Farias VDS, Lima MJA, Ramos TF \& Sousa AML. Produção de Área Foliar e Biomassa e Produtividade do Feijão-caupi sob Regimes Hídricos em Castanhal, Pará. Revista Caatinga, 30(3): 748-759, 2017.

Tenório ARM et al. Mapeamento dos solos da Estação de Pscicultura de Castanhal. 1. ed. Belém: FCAP, 50p. 1999.

Williams JR. Sediment yield prediction with universal equation using runoff energy factor. USDA-ARS S-40: Present and prospective technology for predicting sediment yield and sources. Washington: USDA, 1975.

Wischmeir WH \& Smith DD. Predicting rainfall erosion losses - a guide to conservation planning. Washington, USDA, 58p. (537), 1978.

Biodiversidade Brasileira - BioBrasil.

Edição Temática: Análise de Componentes do Sistema Climático e a Biodiversidade no Brasil

$$
\text { n. 4, } 2021
$$

http://www.icmbio.gov.br/revistaeletronica/index.php/BioBR

Biodiversidade Brasileira é uma publicação eletrônica científica do Instituto Chico Mendes de

Conservação da Biodiversidade (ICMBio) que tem como objetivo fomentar a discussão e a disseminação de experiências em conservação e manejo, com foco em unidades de conservação $e$ espécies ameaçadas.

ISSN: 2236-2886 